\title{
Spontaneous Regression of Methotrexate-related Lymphoproliferative Disorder with T-cell Large Granular Lymphocytosis
}

\author{
Hiroshi Ureshino ${ }^{1}$, Chiho Kadota ${ }^{1}$, Kazuya Kurogi ${ }^{1}$, \\ Masaharu Miyahara ${ }^{1}$ and Shinya Kimura ${ }^{2}$
}

\begin{abstract}
Spontaneous regression of methotrexate-related lymphoproliferative disorders (MTX-LPDs) occurs in some patients after withdrawal of MTX. However, the mechanisms by which MTX withdrawal contributes to the spontaneous regression of MTX-LPDs have not been fully elucidated. We herein show that spontaneous regression of MTX-LPDs is associated with the development of significant and transient T-cell large granular lymphocyte (T-LGL) lymphocytosis induced by MTX withdrawal. Since T-LGLs show strong cytotoxicity, their expansion may contribute to the spontaneous regression of lymphoma. Therefore, the development of TLGL lymphocytosis maybe associated with a favorable prognosis in MTX-LPD patients.
\end{abstract}

Key words: methotrexate-related lymphoproliferative disorders, spontaneous regression, T-cell large granular lymphocytosis, diffuse large B-cell lymphoma

(Intern Med 54: 2235-2239, 2015)

(DOI: 10.2169/internalmedicine.54.4207)

\section{Introduction}

Rheumatoid arthritis (RA) is associated with an increased risk of lymphoproliferative disorders (LPDs). Additionally, the use of immunosuppressive drugs, including methotrexate (MTX), is an independent risk factor for LPDs in patients with RA (1). Spontaneous regression of MTX-related LPD (MTX-LPD) has been reported after withdrawal of MTX (2). Large granular lymphocytes (LGLs), which are characterized by an abundant cytoplasm and fine or coarseazurophilic granules, are classified into T-cell $(\mathrm{T})$ LGLs and natural killer (NK)-LGLs according to their surface markers. T-LGLs express CD3, CD8, CD57, and TIA1, whereas NK-LGLs express CD16 and CD56. Both TLGLs and NK-LGLs are characterized by strong cytotoxicity (3), and expansion of these cells has been reported in a subset of patients with RA, LPDs, or chronic infection; transplantation patients (4); and those with chronic myelogenous leukemia (CML) or Philadelphia chromosome-positive acute lymphocytic leukemia $\left(\mathrm{Ph}^{+} \mathrm{ALL}\right)$ treated with the $\mathrm{ABL}$ tyrosine kinase inhibitor dasatinib (5-7). Furthermore, LGL expansion is associated with a good prognosis in several malignancies $(8,9)$ through its effect on antitumor immune responses. We herein report a case of MTX-LPD achieving complete remission after withdrawal of MTX with significant and transient T-LGL expansion.

\section{Case Report}

A 66-year-old Japanese woman with a 5-year history of RA treated with MTX was admitted to our hospital due to leukopenia and severe thrombocytopenia. Oral MTX had been discontinued 1 week prior to the presentation. On the physical examination, no swollen superficial lymph nodes, splenomegaly, or oral mucosal damage were observed. Computed tomography (CT) imaging revealed no abnormal findings. A hematological analysis indicateda white blood cell (WBC) count of $2.60 \times 10^{9} / \mathrm{L}$ (0\% blasts, $70 \%$ neutrophils, $24 \%$ lymphocytes, and $3 \%$ monocytes), hemoglobin level of

${ }^{1}$ Department of Internal Medicine, Karatsu Red Cross Hospital, Japan and ${ }^{2}$ Division of Hematology, Respiratory Medicine and Oncology, Department of Internal Medicine, Faculty of Medicine Saga University, Japan

Received for publication October 3, 2014; Accepted for publication December 18, 2014

Correspondence to Dr. Masaharu Miyahara, miyahara@karatsu.jrc.or.jp 


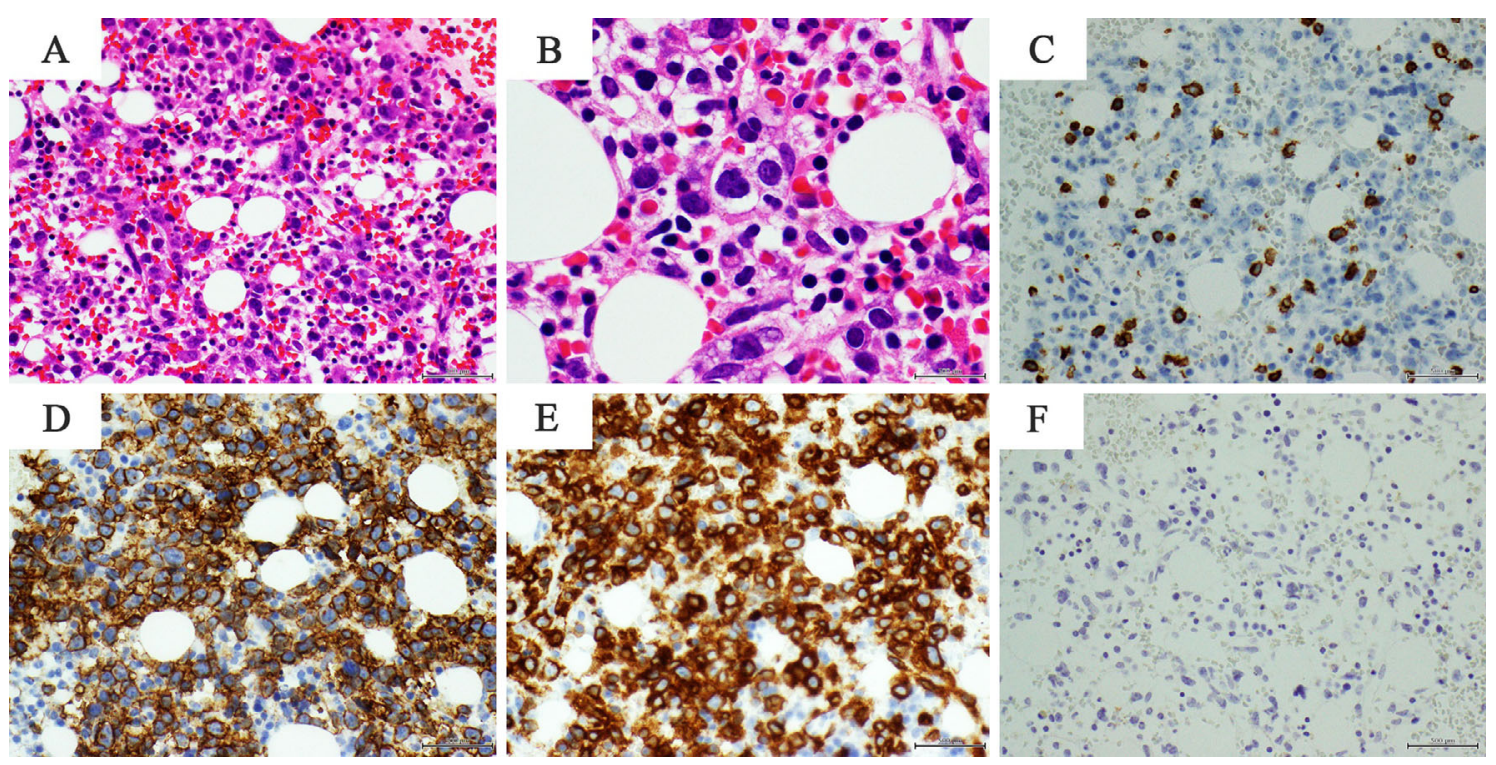

Figure 1. Histological and immunohistochemical findings of the bone marrow. Large-sized lymphoid cells with polymorphic conspicuous nuclear bodies were increased. Lymphoma cells were positive for CD20 and CD79a and negative for CD3 and Epstein-Barr encoding region (EBER). (A) (B) Hematoxylin and Eosin staining. Immunohistochemical staining for CD3 (C), CD20 (D), CD79a (E), and $\operatorname{EBER}(\mathrm{F})$. A, C-F: original magnification, $\times 400 \mathrm{~B} ; \times 1,000$.

$11.6 \mathrm{~g} / \mathrm{dL}$, platelet count of $15 \times 10^{9} / \mathrm{L}$, lactate dehydrogenase (LDH) level of 4,008 IU/L, soluble interleukin 2 receptor (sIL-2R) concentration of 2,920 IU/mL, and no decrease in the vitamin $\mathrm{B}_{12}$ or folic acid levels. Bone marrow (BM) aspiration revealed large lymphoid cells with polymorphic large nuclear bodies. Immunophenotyping and flow cytometric analyses showed abnormal cells expressing CD20 and CD79a but not CD3 (Fig. 1). In the lymphoma cells, southern blot hybridization revealed immunoglobulin heavy-chain gene rearrangement and a flow cytometric analysis showed immunoglobulin kappa light chain restriction (kappa chain/ lambda chain ratio: 13.3). In situ hybridization showed the absence of the Epstein-Barr virus (EBV) small RNA gene (Fig. 1) and quantitative polymerase chain reaction (PCR) was negative for EBV in the serum sample. The patient was diagnosed with MTX-related diffuse large B-cell lymphoma (DLBCL) without EBV activation. After admission, MTX discontinuation was maintained and the patient was placed under observation. The number of WBCs increased significantly to $42.5 \times 10^{9} / \mathrm{L}$ on day 14 after withdrawal of MTX (Fig. 2), and most WBCs were lymphocytes $\left(38.7 \times 10^{9} / \mathrm{L}\right)$. The lymphocytes in the peripheral blood (PB) had abundant cytoplasm and azurophilic granules, indicating that they were LGLs. The LDH level was 2,261 IU/L. A flow cytometric analysis showed predominantly $\mathrm{CD}^{+} \mathrm{CD}^{+} \mathrm{T}$-cells in the PB. BM aspiration showed that the proportion of lymphoma cells decreased from 33.6 to $0.4 \%$; the lymphocytes increased and showed abundant cytoplasm and azurophilic granules. An immunophenotyping analysis showed that the lymphocytes in the $\mathrm{BM}$ expressed $\mathrm{CD} 3, \mathrm{CD} 4, \mathrm{CD} 8$, and TIA-1, but were negative for CD20 and CD56 (Fig. 3). Furthermore, TIA-1 was expressed in $\mathrm{CD}^{+} \mathrm{T}$-cells, but not in
$\mathrm{CD} 4^{+} \mathrm{T}$-cells. The predominant phenotype was $\mathrm{CD}^{+} \mathrm{CD}^{+} \mathrm{T}-$ lymphocytes, as determined by flow cytometry (gating for lymphocytes: CD3, 93\%; CD4, 37.5\%; and CD8, 58.8\%). A southern blot analysis of the BM and $\mathrm{PB}$ cells revealed the presence of monoclonal T-cell receptor (TCR)-C $\beta$ gene rearrangement (Fig. 3). The LDH levels decreased gradually immediately prior to the occurrence of T-LGL lymphocytosis. The number of T-LGLs decreased rapidly within 1 week, and the platelet count simultaneously recovered (Fig. 2). After the appearance of T-LGLs, no increase in the uric acid or potassium levels, which would suggest tumor lysis syndrome, occurred (Fig. 2). BM aspiration and positron emission tomography (PET)/CT scanning were performed 1 month after admission. The results of the BM aspiration showed the disappearance of the lymphoma cells from the $\mathrm{BM}$, and a whole-body PET-CT scan showed no abnormal 2- $\left[{ }^{18} \mathrm{~F}\right]$-fluoro-2-deoxy-D-glucose (FDG) uptake (Fig. 4). These findings indicated that the patient achieved complete remission within 1 month after the expansion of transient TLGLs induced by MTX withdrawal. Complete remission was sustained for 2 months.

\section{Discussion}

The spontaneous regression of MTX-LPDs occurs in 59\% of patients after MTX withdrawal (10). Therefore, the withdrawal of MTX is the first choice of treatment for patients with MTX-LPD (2). The incidence of spontaneous regression is dependent on the histological features and EBV infection status. Patients showing spontaneous regression have a significantly higher rate of EBV positivity ( $85 \%$ vs. $50 \%$ ) and a lower frequency of DLBCL, as compared to other 


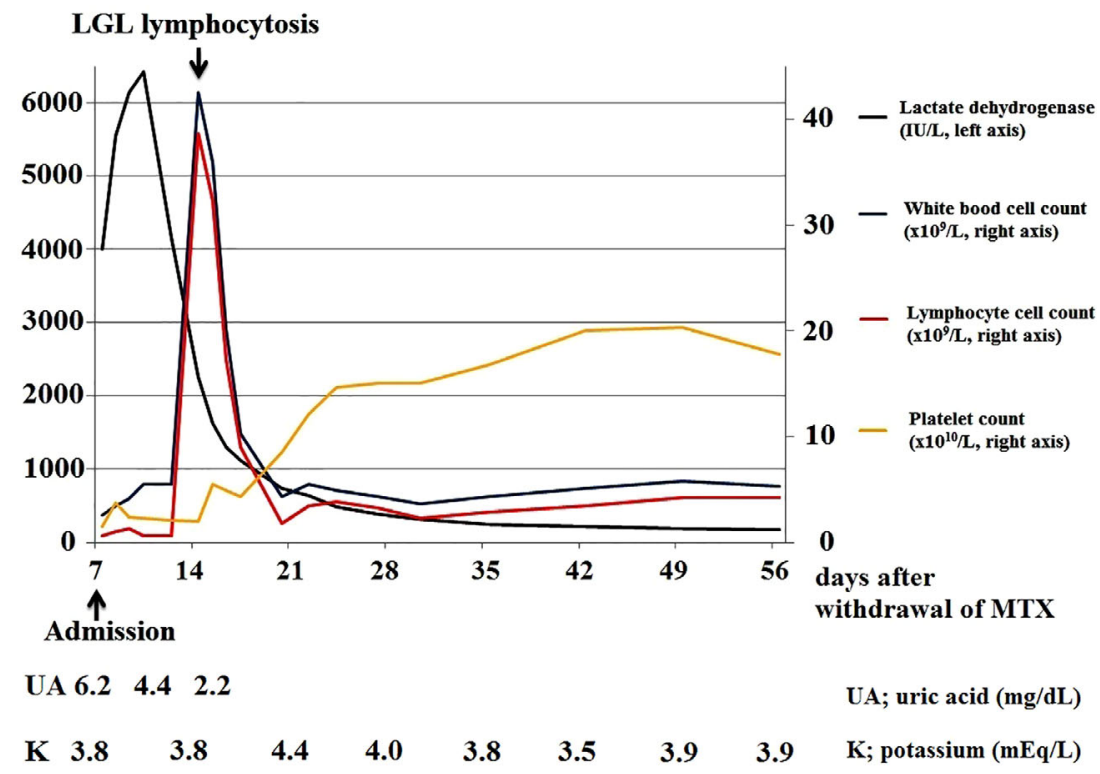

Figure 2. Clinical course. T cell large granular lymphocyte (T-LGL) lymphocytosis occurred Day 14 after withdrawal of MTX. Lactate dehydrogenase level decreased gradually from Day 12 after withdrawal of MTX. T-LGL rapidly decreased within 1 week and platelet count was simultaneously recovered. After appearance of T-LGLs, it was not occurred elevation of uric acid and potassium level.
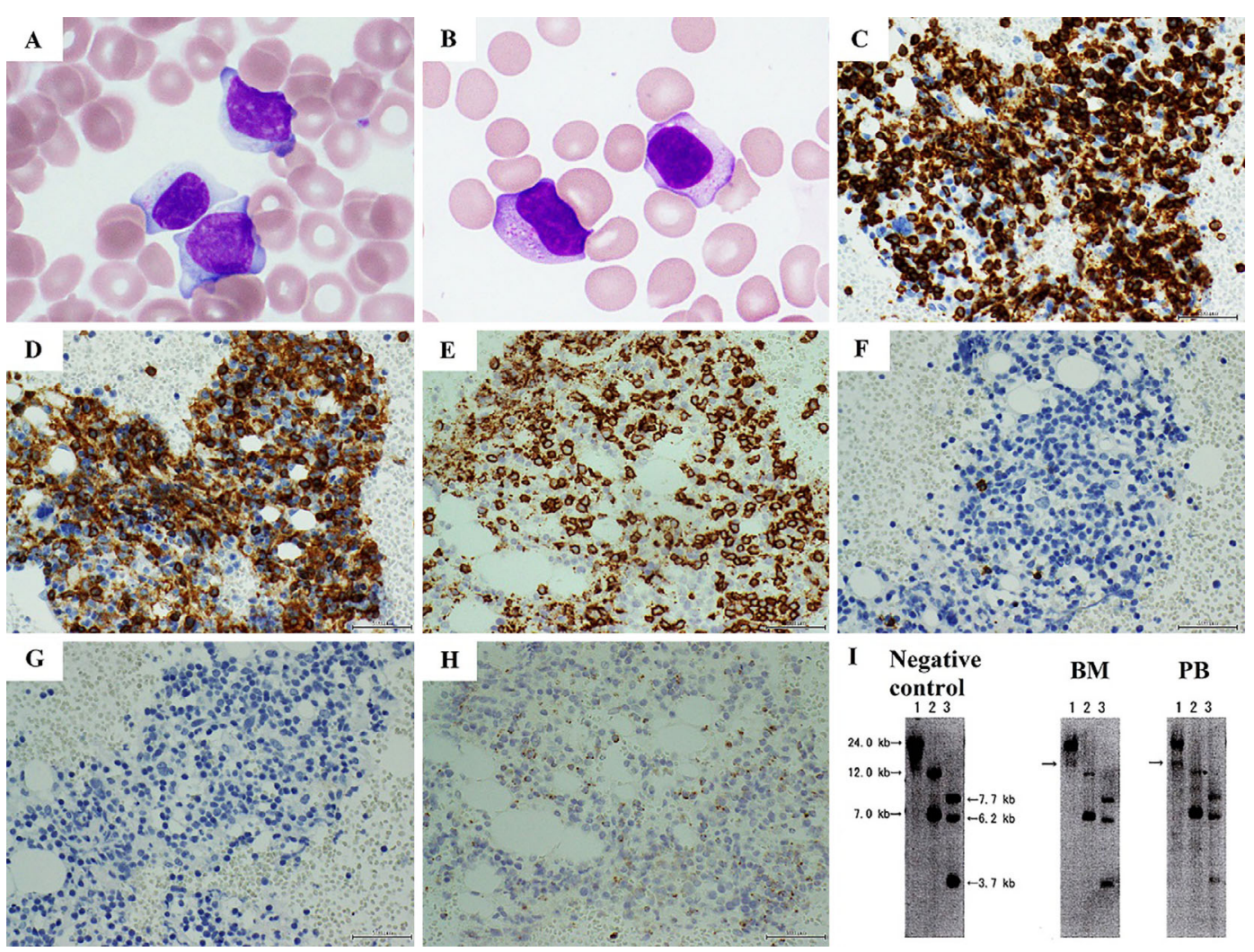

Figure 3. Histological and immunohistochemical findings of the peripheral blood and bone marrow Wright stained peripheral blood smear (A) and bone marrow aspirate smear (B) showing large granular lymphocytes (A, B: original magnification, 1,000×). The lymphocytes were positive for $\mathrm{CD3}(\mathrm{C})$, CD4 (D), CD8 (E), and TIA-1 (H) whereas they were negative for CD20 (F) and CD56 (G). (C-F: original magnification, 400 $\times$ ) I; Southern blots showing clonal TCR-C $\beta$ gene rearrangement in mononuclear cells derived from the bone marrow (BM) and peripheral blood (PB). The rearranged bands were identical in size after digestion with BamHI and Hind III restriction enzymes (restriction enzymes: lane 1, BamHI I; lane 2, EcoRV V; lane 3, Hind III). 

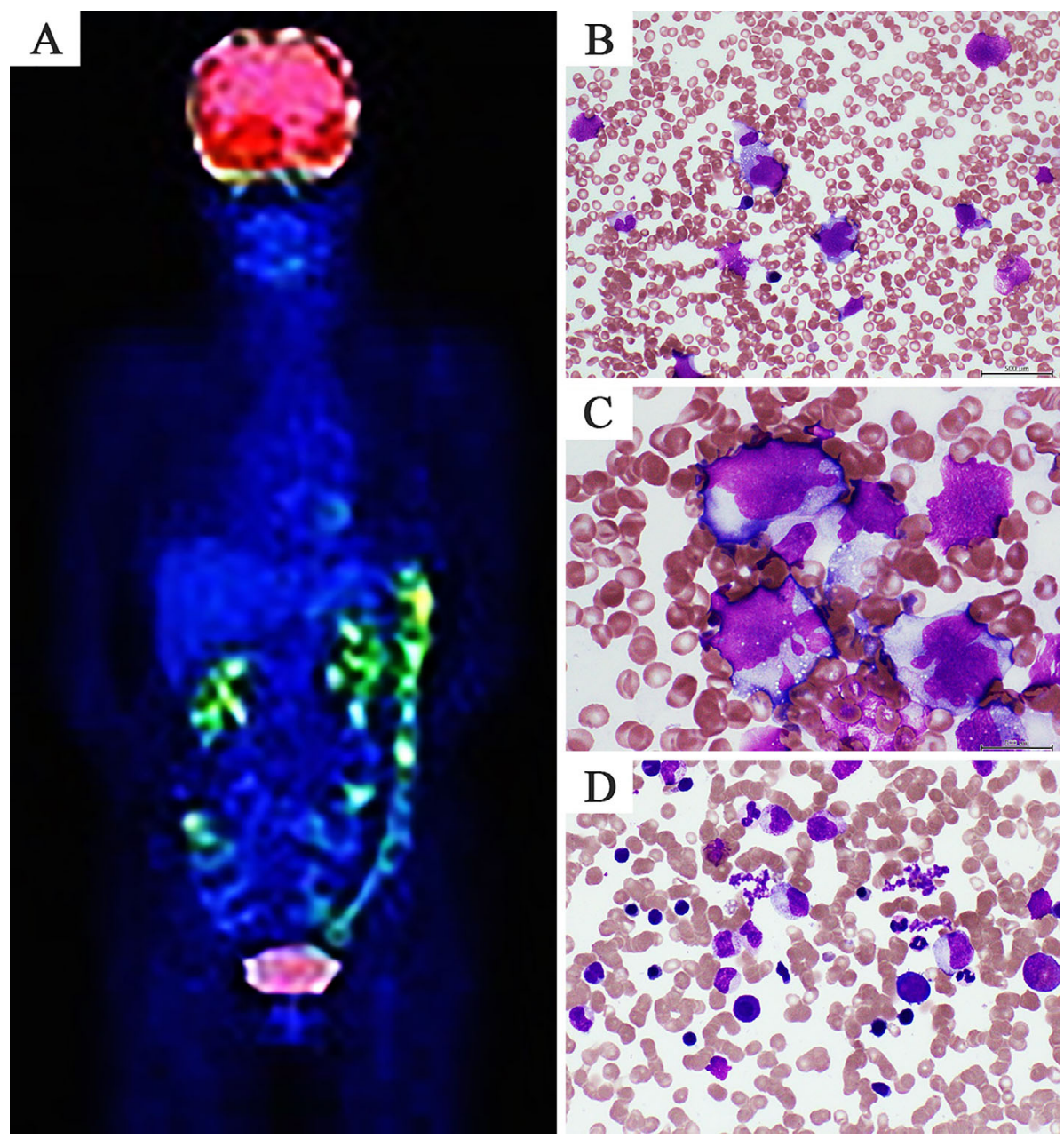

Figure 4. PET-CT and histology after regression of MTX-LPDs (A) Positron emission tomography/ computed tomography (PET/CT) imaging. No abnormal 2-[18F]-fluoro-2-deoxy-D-glucose (FDG) uptake was observed in the whole body. Wright stained bone marrow aspirate smear showing increased lymphoma cells before T-LGL expansion. Wright stained bone marrow aspirate smear showing the disappearance of lymphoma cells after T-LGL expansion (B, D: original magnification, 400x; $C: 1,000 \times)$. There were few CD20 and CD79a positive lymphocytes and there was no detection of immunoglobulin heavy-chain gene rearrangement by southern blot hybridization and immunoglobulin kappa light chain restriction by flow cytometry.

subtypes (39\% vs. 78\%), than those without regression (10). Because the EBV result was negative and the disease subtype was DLBCL in the present case, spontaneous regression was thus considered unlikely. LGL lymphocytosis is associated with several diseases, including RA (5-7). In the present case, LGL lymphocytosis had not been detected before MTX withdrawal.

Although MTX-LPDs can occur concurrently with T-LGL lymphocytosis, the coexistence of LPDs and T-LGL lymphocytosis in the same patient is uncommon. Disorders such as chronic lymphocytic leukemia (11), hairy cell leukemia (12), splenic marginal zone lymphoma (13), monoclonal B-cell lymphocytosis (14), and splenic diffuse red pulp small B-cell lymphoma (15) have been reported in association with LPDs. Increases of T-LGLs are associated with chronic infection and allograft transplantation, most likely due to chronic antigen stimulation and the subsequent ex- pansion of antigen-activated cytotoxic T-cells. Similarly, the proliferation of T-LGLs maybe induced by the antigen stimulation associated with the function of LPD cells. In this manner, MTX-LPDs and T-LGL lymphocytosis could be closely related.

In the present case, a possible infiltration of $\mathrm{CD}^{+} \mathrm{T}$-cells in the $\mathrm{BM}$ was seen (Fig. 3). $\mathrm{CD}^{+} \mathrm{T}$-cells and $\mathrm{CD} 8^{+} \mathrm{T}$-cells are the major populations of T-cells in the $\mathrm{PB}$ and BM. These cells are related to each other, and activated $\mathrm{CD} 8^{+} \mathrm{T}-$ cells induce $\mathrm{CD} 4^{+} \mathrm{T}$-cell expansion (16). In the present case, $\mathrm{CD}^{+} \mathrm{CD}^{+} \mathrm{T}$-LGLs induced $\mathrm{CD} 4^{+} \mathrm{T}$-cells, resulting in an increase of $\mathrm{CD}^{+} \mathrm{T}$-cells in the $\mathrm{BM}$. The infiltration of $\mathrm{CD} 4^{+}$ T-cells in the BM was thought to be associated with the presence of T-LGLs and not with the clinical course of the disease.

The plasma levels of interleukin (IL)-6, which are higher in RA patients than in healthy individuals, are reduced by 
MTX treatment in RA patients (17). The withdrawal of MTX raises the plasma levels of IL-6, and increased IL-6 activates signal transducer and activator of transcription 3 (STAT3), thus leading to LGL lymphocytosis through the inhibition of apoptosis (18).

Major histocompatibility complex haplotype associations in RA and LGL lymphocytosis are secondary to allelic association with human leukocyte antigen (HLA)-DRB1* 0401 (19). LGL lymphocytosis is also related to the T-cell receptor $\beta$-chain (TRB) gene repertoire (20). In the present case, the patient may have had alleles prone to LGL lymphocytosis and a TRB gene repertoire associated with DLBCL antigen recognition; MTX withdrawal may have induced an increase in the plasma level of IL-6 and activation of STAT3, resulting intransient T-LGL lymphocytosis. Spontaneous regression may have occurred as a result of the cytotoxicity of T-LGL expansion. Large granular lymphocytosis is caused by the proliferation of reactive or neoplastic lymphocytes, similar to large granular lymphocyte leukemia (21). LGL expansion that disappears with no treatment is presumed to be reactive.

To the best of our knowledge, there are no previously reported cases of significant and transient T-LGL lymphocytosis associated with the spontaneous regression of MTXrelated LPDs, such as was observed in the present case. The occurrence of T-LGL lymphocytosis is a favorable prognostic factor in patients with MTX-related LPDs, as well as in CML patients treated with dasatiniband in transplant patients. Further investigation of MTX-related LPDs and LGL is necessary to confirm the present findings.

\section{The authors state that they have no Conflict of Interest (COI).}

\section{Acknowledgement}

We greatly thank Dr. Kohichi Ohshima M.D., Ph.D. (Department of Pathology, School of Medicine, Kurume University, Kurume, Japan) for assistance with the pathological diagnosis.

\section{References}

1. Wolfe F, Michaud K. Lymphoma in rheumatoid arthritis: the effect of methotrexate and anti-tumor necrosis factor therapy in 18,572 patients. Arthritis Rheum 50: 1740-1745, 2004.

2. Salloum E, Cooper DL, Howe G, et al. Spontaneous regression of lymphoproliferative disorders in patients treated with methotrexate for rheumatoid arthritis and other rheumatic diseases. J Clin Oncol 14: 1943-1949, 1996.

3. Gastl G, Niederwieser D, Marth C, et al. Human large granular lymphocytes and their relationship to natural killer cell activity in various disease states. Blood 64: 288-295, 1984.

4. Zambello R, Semenzato G. Large granular lymphocytosis. Haematologica 83: 936-942, 1998.

5. Mustjoki S, Ekblom M, Arstila TP, et al. Clonal expansion of T/ NK-cells during tyrosine kinase inhibitor dasatinib therapy. Leuke- mia 23: 1398-1405, 2009.

6. Kim DH, Kamel-Reid S, Chang H, et al. Natural killer or natural killer/T cell lineage large granular lymphocytosis associated with dasatinib therapy for Philadelphia chromosome positive leukemia. Haematologica 94: 135-139, 2009.

7. Nagata Y, Ohashi K, Fukuda S, Kamata N, Akiyama H, Sakamaki H. Clinical features of dasatinib-induced large granular lymphocytosis and pleural effusion. Int J Hematol 91: 799-807, 2010.

8. Pandolfi F, Loughran TP, Starkebaum G, et al. Clinical course and prognosis of the lymphoproliferative disease of granular lymphocytes. A multicenter study. Cancer 65: 341-348, 1990.

9. Kim D, Al-Dawsari G, Chang H, et al. Large granular lymphocytosis and its impact on long-term clinical outcomes following alloSCT. Bone Marrow Transplant 48: 1104-1111, 2013.

10. Ichikawa A, Arakawa F, Kiyasu J, et al. Methotrexate/iatrogenic lymphoproliferative disorders in rheumatoid arthritis: histology, Epstein-Barr virus, and clonality are important predictors of disease progression and regression. Eur J Haematol 91: 20-28, 2013.

11. Martinez A, Pittaluga S, Villamor N, et al. Chronal T-cell population and increased risk for cytotoxic T-cell lymphomas in B-CLL patients: clinicopathologic observations and molecular analysis. Am J Surg Pathol 28: 849-858, 2004.

12. Xie XY, Sorbara L, Kreiman RJ, Fukushima PI, Kingma DW, Stetler-Stevenson M. Development of lymphoproliferative disorder of granular lymphocytes in association with hairy cell leukemia. Leuk Lymphoma 37: 97-104, 2000.

13. Lima M, Goncalves C, Marques L, et al. Association of CD4+/ CD56+/CD57+/CD8+(dim) large granular lymphocytic leukemia, splenic B-cell lymphoma with circulating villous lymphocytes, and ideopathic erythrocytosis. Ann Hematol 80: 685-690, 2001.

14. Howard MT, Bejanyan N, Maciejewski JP, His ED. T/NK large granular lymphocyte leukemia and coexisting monochronal B-cell lymphocytosis-like proliferations. An unrecognized and frequent association. Am J Clin Pathol 133: 936-941, 2010.

15. Setoodeh R, Zhang L, Shao H. Concurrent splenic diffuse red pulp small B-cell lymphoma and benign clonal proliferation of Tcell large granular lymphocytosis. Leukemia Res 36: 103-106, 2012.

16. Kitchen SG, Berthou C, Patel VP, Kitchen CM, Miceli MC, Zack JA. Activation of $\mathrm{CD} 8 \mathrm{~T}$ cells induces expression of $\mathrm{CD} 4$, which functions as a chemotactic receptor. Blood 99: 207-212, 2002.

17. Nishina N, Kaneko Y, Kameda H, Kuwana M, Takeuchi T. Reduction of plasma IL- 6 but not TNF- $\alpha$ by methotrexate in patients with early rheumatoid arthritis: a potential biomarker for radiographic progression. Clin Rheumatol 32: 1661-1666, 2013.

18. Schade AE, Wlodarski MW, Maciejewski JP. The role of JAKSTAT and PI3K signaling in leukemic large granular lymphocytes. Cell Cycle 5: 2571-2574, 2006.

19. Coakley G, Brooks D, Iqbal M, et al. Major histocompatility complex haplotypic associations in Felty's syndrome and large granular lymphocyte syndrome are secondary to allelic association with HLA-DRB1*0401. Rheumatology 39: 393-398, 2000.

20. Papalexandri A, Stalika E, Iskas M, et al. Molecular evidence for repertoire skewing of $\mathrm{T}$ large granular lymphocyte proliferation after allogeneic hematopoietic SCT: report of two cases. Bone Marrow Transplant 48: 1260-1261, 2013.

21. Cornec D, Berthou C, Douet-guilbert N, Saraux A. Long-term remission of T-cell large granular lymphocyte leukemia associated with rheumatoid arthritis after rituximab therapy. Blood 122: 1583-1586, 2013.

(C) 2015 The Japanese Society of Internal Medicine http://www.naika.or.jp/imonline/index.html 